\title{
Somatosensory prior entry assessed with temporal order judgments and simultaneity judgments
}

\author{
Mark J. Yates • Michael E. R. Nicholls
}

Published online: 13 April 2011

(C) Psychonomic Society, Inc. 2011

\begin{abstract}
Attention is central to perception, yet a clear understanding of how attention influences the latency of perception has proven surprisingly elusive. Recent research has indicated that spatially attended stimuli are perceived earlier than unattended stimuli across a range of sensory modalitiesan effect termed prior entry. However, the method commonly used to measure this, the temporal order judgment (TOJ) task, has been criticized as susceptible to response bias, despite deliberate attempts to minimize such bias. A preferred alternative is the simultaneity judgment (SJ) task. We tested the prior-entry hypothesis for somatosensory stimuli using both a TOJ task (replicating an earlier experiment) and an SJ task. Prior-entry effects were found for both, though the effect was reduced in the SJ task. Additional experiments (TOJ and SJ) using visual cues established that the earlier perception of cued tactile targets does not result from intramodal sensory interactions between tactile cues and targets.
\end{abstract}

Keywords Time perception - Temporal - Order judgment . Simultaneity judgment - Spatial attention · Prior entry . Somatosensory

The perceived timing of events does not always match the reality of their timing. An excellent example of this dissociation comes from the phenomenon of prior entry, whereby spatially attended stimuli are perceived earlier than otherwise identical unattended stimuli. For example, when

M. J. Yates $(\square)$

Department of Psychology, University of Melbourne,

Parkville, Victoria 3010, Australia

e-mail: mjyates@unimelb.edu.au

M. E. R. Nicholls

School of Psychology, Flinders University,

Adelaide, South Australia, Australia asked to judge the timing of an event, participants will typically judge that event to occur earlier if they are attending to the spatial location at which the event occurs. Prior entry has been demonstrated for stimuli occurring in the visual (Shore, Spence, \& Klein, 2001), auditory (Kanai, Ikeda, \& Tayama, 2007), and somatosensory (Yates \& Nicholls, 2009) modalities. For visual and somatosensory stimuli, this effect has been demonstrated both when attention is manipulated involuntarily, by means of spatially uninformative peripheral exogenous cues, and voluntarily, by means of spatially informative, centrally presented symbolic arrow cues. Taken together, these findings constitute an important addition to our understanding of the temporal dynamics of attention. Investigating the influence of attention on perceived timing also offers an opportunity to shed light on a central question in neuroscience-namely, how subjective time is represented in the brain (e.g., Dennett \& Kinsbourne, 1992; Eagleman et al., 2005). The research can therefore address an influential idea expressed by Köhler: "Experienced order in time is always structurally identical with a functional order in the sequence of correlated brain processes" (1947, p. 62).

Although the prior-entry paradigm is well established, a question mark hangs over the reported effects of attention on perceptual latency, stemming from two prominent concerns. First, the method traditionally used to assess shifts in perceived timing in these experiments, the temporal order judgment (TOJ) task, has increasingly been queried (e.g., Schneider \& Bavelier, 2003; Shore \& Spence, 2005; Zampini, Shore, \& Spence, 2005). The concern is that estimates of perceived timing derived from this method might largely reflect response bias effects rather than genuine perceived-timing effects. This concern persists despite improvements to the TOJ task, which have eliminated its most obvious limitations (Shore \& Spence, 2005; Shore et al., 2001; Yates \& Nicholls, 2009). Fortunately, the simultaneity judgment (SJ) task has 
been put forward to overcome this response bias problem. This method is gaining traction among researchers in the field (e.g., Santangelo \& Spence, 2008; Schneider \& Bavelier, 2003; Zampini, Shore, \& Spence, 2005; for a full account of the SJ task and a rationale for its use in prior-entry studies, refer to Schneider \& Bavelier, 2003). It is also worth noting that some have argued that the SJ and TOJ tasks may measure different aspects of temporal perception (Allan, 1975; Jaśkowski, 1991; van Eijk, Kohlrausch, Juola, \& van de Par, 2008).

The second concern about the reported effects of attention on perceptual latency is that the earlier perception of attended stimuli can be attributed to low-level sensory interactions between the target stimuli and the cues used to orient attention, rather than being a consequence of attention per se (see Schneider \& Bavelier, 2003; Spence \& Parise, 2010). This second concern applies regardless of which method is used to assess perceived timing, but it is only relevant for experiments in which attention is manipulated exogenously by peripheral cues presented close to the target stimuli.

In this study, we addressed these concerns in an investigation of somatosensory prior entry, using a two-by-two matrix of four experiments. The dimensions of the matrix were task type (TOJ_-first two experiments - or SJ_last two experiments) and cue type (tactile or visual cues). Throughout all of the experiments, the target stimuli were always two tactile stimuli (i.e., participants' judgments always concerned the relative timing of two tactile stimuli, one of which was attended and one of which was unattended). In Experiment 1, we replicated our original somatosensory/tactile prior-entry finding (Yates \& Nicholls, 2009), with one key improvement - the introduction of eye movement monitoring and the removal of trials with deviations of gaze from central fixation. This allowed us to rule out the possibility that observed perceived timing shifts were caused by overt rather than covert shifts in spatial attention. In Experiment 2, we replaced the spatially nonpredictive tactile cues of Experiment 1 with visual cues, to determine whether the attended targets would still be perceived earlier in circumstances where low-level sensory interactions between cues and targets were precluded. Experiments 3 and 4 were essentially identical to the first two experiments, except that a simultaneity judgment was used instead of a temporal order judgment. All experiments involved embedded reaction time probe tasks as an independent measure that spatial cuing had manipulated spatial attention as intended (see Cairney, 1975; Shore \& Spence, 2005; Spence, Shore, \& Klein, 2001; Vanderhaeghen \& Bertelson, 1974, for discussions of why this is important).

\section{Experiment 1}

Experiment 1 tested the effect of spatially uninformative tactile exogenous cuing (i.e., brief cues to the left, to the right, or to both hands) on the time at which two subsequent tactile stimuli (one delivered to each hand) were perceived. This experiment is essentially a replication of Experiment 1 in Yates and Nicholls (2009), but with one important difference: the inclusion of eye movement monitoring and the exclusion of all trials on which participants' gaze deviated from central fixation.

Method

\section{Participants}

Sixteen healthy students took part in this experiment in return for course credit at the University of Melbourne. The complete data sets (both the reaction time and TOJ data) were excluded for 5 participants due to a failure to achieve an accuracy threshold, which was $70 \%$ (or greater) correct for the TOJs. All of the remaining participants (4 male, 7 female) were strongly right-handed according to the Edinburgh Inventory (Oldfield, 1971). This experiment and the other experiments in this study received approval from the University of Melbourne Ethics Committee, and all participants gave informed, written consent prior to participating in the study.

\section{Apparatus and stimuli}

The experiment was conducted in a darkened, soundattenuated room. Participants sat at a desk facing a monitor interfaced with a PC computer, with their chins placed in a chinrest. Figure 1 shows two vertical stands with hand grips $600 \mathrm{~mm}$ apart. The hands were placed on the ipsilateral hand grips and positioned so that the index fingers of both hands were located directly above the thumbs of the same

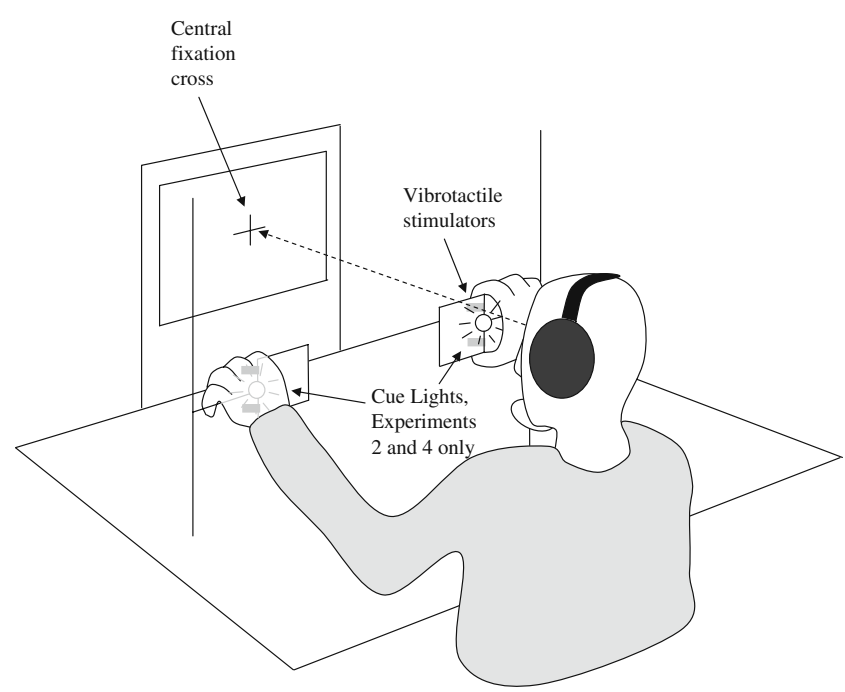

Fig. 1 Schematic view of the apparatus and of participants' posture for all four experiments. Response pedals were located under the desk 
hand. The vertical separation between the tip of the index finger and the tip of the thumb was $100 \mathrm{~mm}$. Plastic vibrotactile stimulators ("tappers"; C-2 tactors, Engineering Acoustics, Inc.) were secured to the thumb, index finger, ring finger, and fifth (little) finger of each hand, with the vibrating surfaces of the device in contact with the finger/ thumb pads (see Fig. 2). Activation of the tappers was controlled by an interface card with a millisecond timer (DCM-16 Digital Interface Card, Blue Chip Technology). A video camera displayed participants' eye movements on a monitor visible to the experimenter.

Three different classes of stimuli were administered during the experiment: cues, TOJ taps (target taps), and reaction time probes (see Fig. 2). Cues consisted of brief (20-ms) bursts of vibrotactile stimulation $(250 \mathrm{~Hz})$ delivered via activation of the tappers secured to the fifth finger of the left hand, the right hand, or both hands. TOJ taps consisted of a brief (10-ms) vibration of the tappers secured to the index finger of one hand and to the thumb of the other hand. These brief bursts of vibration from the tappers resulted in a sensation similar to receiving a light tap on the stimulated finger. Reaction time probes consisted of three pulses of vibration, with each pulse lasting $40 \mathrm{~ms}$ and being separated by $40 \mathrm{~ms}$ (total duration of $200 \mathrm{~ms}$ ) delivered by tappers secured to the fourth finger of either hand. This was experienced by participants as a rough tactile "buzz" with discernible pulses. Throughout the experiment, white noise at $70 \mathrm{~dB}$ was presented via headphones to mask any noise made by the operation of the tappers and any extraneous noise. Additionally, the vibration of the tappers was not visually detectable.

\section{Procedure}

Figure 3 illustrates the sequence of stimuli during a trial. A trial consisted of the presentation of a white central fixation cross against a black background on the computer screen, which remained present for the duration of the trial. After $300 \mathrm{~ms}$, a cue to attract attention was delivered, either to the left, to the right, or to both sides. The neutral cue condition (alerted condition without spatial information) provided a control condition, which should be intermediate between the upper- and lower-tap cuing conditions, while holding constant the alerting effect due to cuing and the orienting of attention in the temporal dimension (Coull \& Nobre, 1998) across all three conditions. The cues were randomly presented with equal frequency and were spatially uninformative (i.e., the location of the cue provided no information about the location of the subsequently presented target).

TOJ trials In five out of six trials, $80 \mathrm{~ms}$ after cue offset (100 ms after cue onset), two TOJ taps were administered, one to each hand, separated by a variable interval. The taps were always presented in pairs of different elevations - that is, one tap to the index finger (upper elevation) of one hand and one tap to the thumb (lower elevation) of the other hand. One tap was presented to the left hand and one to the right hand for all trials. In half of the trials, a digit on the

\begin{tabular}{llll}
\multicolumn{1}{|l}{ Experiment 1} & Stimulus Type & Duration \\
\hline Thumb (A) & Target Tap (Lower Tap) & $10 \mathrm{~ms}$ \\
Index Finger (B) & Target Tap (Upper Tap) & $10 \mathrm{~ms}$ \\
Fourth Finger (C) & Reaction Time Probe & $200 \mathrm{~ms}$ \\
Fifth Finger (D) & Exogenous Cue Tap & $20 \mathrm{~ms}$
\end{tabular}

Experiment 2

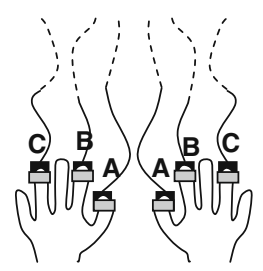

\begin{tabular}{|lll|}
\hline Location & Stimulus Type & Duration \\
\hline Thumb (A) & Target Tap (Lower Tap) & $10 \mathrm{~ms}$ \\
Index Finger (B) & Target Tap (Upper Tap) & $10 \mathrm{~ms}$ \\
Fourth Finger (C) & Reaction Time Probe & $200 \mathrm{~ms}$ \\
Secured to Hand Grip & Exogenous Cue Light & $20 \mathrm{~ms}$ \\
\hline
\end{tabular}

Fig. 2 Placement of stimuli for Experiments 1 and 2. The diagrams are schematic only and do not represent the actual posture of the hands during the experiments. Tappers (here shown in black) were secured to the fingers and thumbs of each hand by fasteners (shown in gray) 
Fig. 3 Sample sequence of events for a single trial in Experiments 1 and 2. The diagram is schematic only and does not represent the actual posture of the hands during the experiment. Cuing was either tactile (Exp. 1) or visual (Exp. 2). The experimental stimuli were identical for Experiments 1 and 2. The central fixation cross was displayed until a response was made
Stage 1 - Cuing

Experiment 1 (Tactile Cuing)

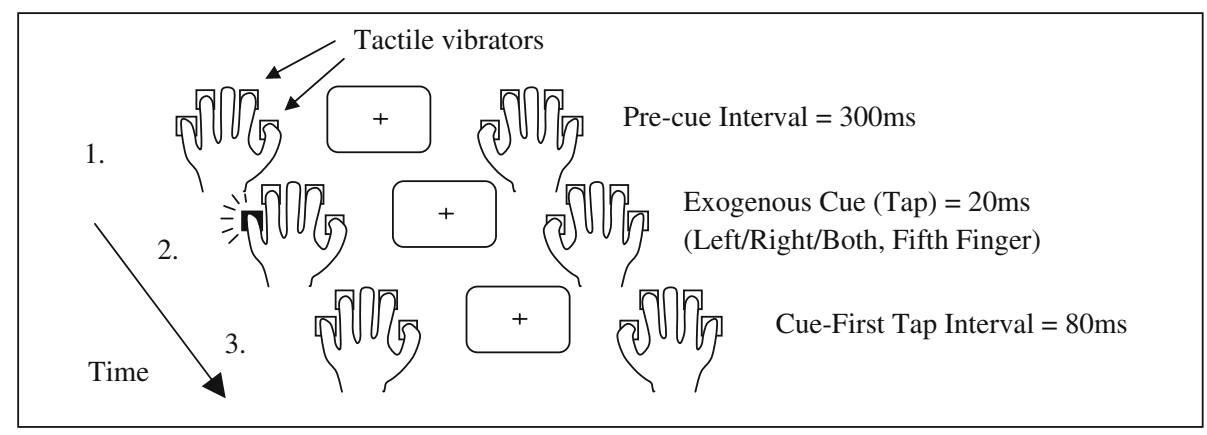

Experiment 2 (Visual Cuing)

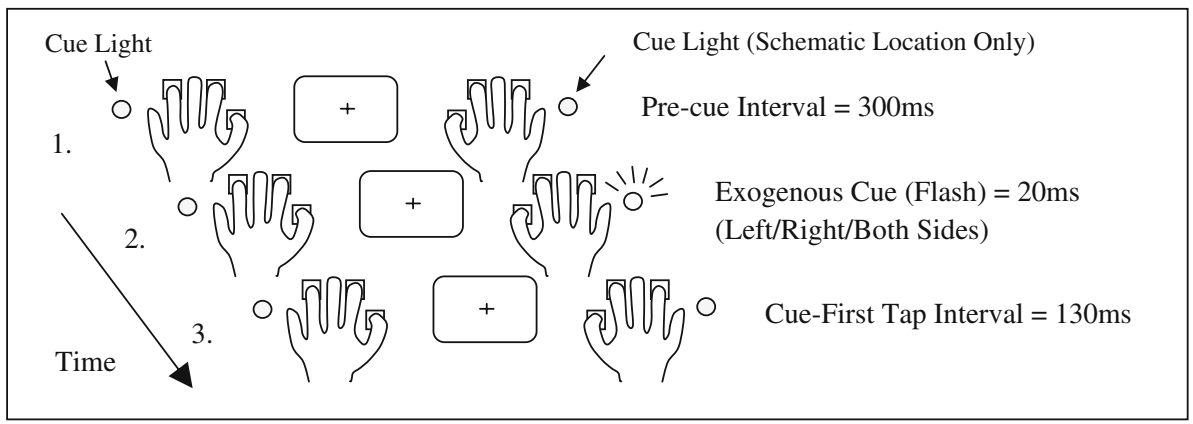

Stage 2 - Experimental Stimuli (Experiments 1 and 2)

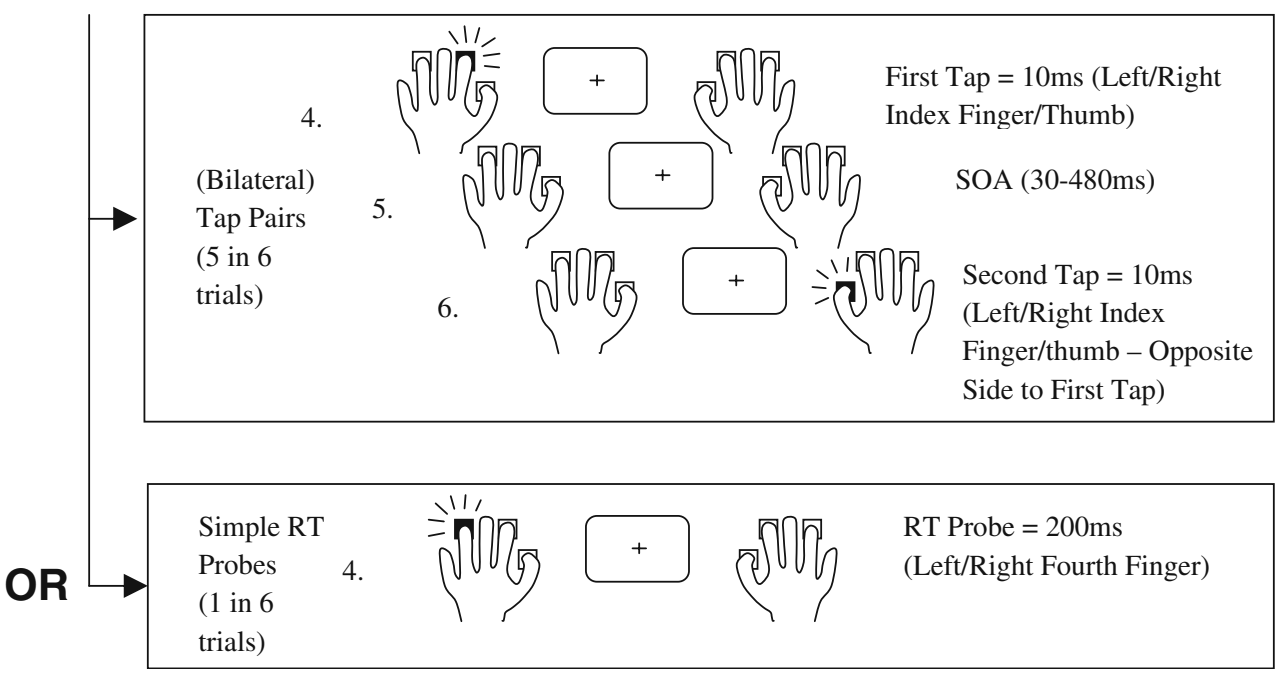

right hand (index finger or thumb) was stimulated first, and on the remaining trials a digit on the left hand was stimulated first. The taps were separated by one of 10 randomly assigned intervals ranging from -480 to $+480 \mathrm{~ms}$ $(-480,-270,-180,-90,-30,+30,+90,+180,+270$, or $+480 \mathrm{~ms}$; positive values indicate that the upper-elevation tap was presented first). The cue duration of $20 \mathrm{~ms}$ plus the 80-ms interval prior to the first experimental tap- $100 \mathrm{~ms}$ in total from cue onset to first stimulus onset-was the same used in Yates and Nicholls (2009), which, in turn, was chosen to closely approximate the total duration separating cue onset from the first stimulus onset in Shore et al. (2001). In that study, which investigated visual prior entry, the exogenous cue was displayed for $45 \mathrm{~ms}$, followed by a 60-ms interval to the first experimental stimulus.

Participants judged the order of the two taps. Half of the participants were instructed to make unspeeded "which tap first?" judgments (i.e., "was the first tap an upper/index finger tap or lower/thumb tap?"). The remaining half made the converse "which tap second?" judgments. The side to 
which the taps were delivered was irrelevant to the task. The manipulation of spatial attention (left-right, horizontal dimension) was therefore orthogonal to the response dimension (upper-lower, vertical dimension). This eliminated the possibility of simple/first-order response bias (i.e., participants reporting the side to which they had been instructed to attend if the task had been simply to report which side was stimulated first). Participants responded in a forced choice manner by releasing one of two pedals located under the toe and heel of the right foot. Release of the toe pedal indicated that the upper tap was judged first (or second, depending on task instructions). Release of the heel pedal indicated that the lower tap was judged first (or second, depending on the task). This mapping of pedal responses to tap elevations was kept constant throughout the experiment and was the most spatially intuitive, since participants perceived the toe pedal as the "upper" pedal with respect to their bodies, and the heel pedal as the "lower" pedal.

Reaction time probe trials In one of six trials, $80 \mathrm{~ms}$ after cue offset (100 ms after cue onset) reaction time probes were delivered to the ring finger of either the left or the right hand. All participants were instructed to respond to this stimulus by releasing the pedal located under their left foot as quickly as possible, irrespective of the hand to which the stimulus was delivered. The reaction time was recorded.

General information Participants were told to focus on accuracy, with no instruction to give rapid responses, unless responding to a reaction time probe. The intertrial interval was $1,500 \mathrm{~ms}$, and feedback regarding accuracy was given at the conclusion of each experimental block.

Participants completed three blocks of 120 trials each $\left(360\right.$ trials $\left.^{1}\right)$. A practice block of 72 trials was delivered prior to testing. In this block, relatively large stimulus onset asynchronies (180 and $270 \mathrm{~ms}$ ) between the two taps were used to facilitate learning of the task. Feedback was given

\footnotetext{
${ }^{1}$ In the Method section for Experiment 1, it is reported that participants completed three blocks with 120 trials in each block (360 trials in total). In fact, for each experiment, all participants completed six blocks with 120 trials in each block (720 trials in total). For three of these blocks, participants adopted a default, uncrossed arms posture. In this posture, the left hand was located in the left hemispace, and the right hand in the right hemispace. Only the data from these three blocks are reported in this study (for each experiment). Participants also completed three blocks while adopting a crossed-arms posture, with the left hand located in the right hemispace and the right hand located in the left hemispace. No prior-entry effects were detected in any of the experiments when participants adopted this crossed-arms posture. However, simple cuing effects (faster reaction times to cued vs. uncued tactile probes) were observed in this posture for the two experiments in which tactile cuing was employed (Exps. 1 and 3).
}

after every trial in the practice block. The practice block was repeated until a performance hurdle of $70 \%$ correct was achieved.

Participants' eye movements were monitored continuously by the experimenter throughout the experiment. Trials involving any deviation of gaze from central fixation were eliminated in real time and substituted with a new trial, which was identical to the trial it replaced (i.e., same cue condition, same SOA between taps).

Results and discussion

\section{Cuing effects}

Mean reaction times to the intermittently presented tactile probe stimuli were analyzed with a within-participants ANOVA with a single factor, Cue Type, with three levels: valid, neutral, and invalid. A validly cued tactile probe was one presented to the same hand as the tactile cue. The slowest $10 \%$ of reaction times were removed prior to analysis for this, and all remaining, experiments. Effect sizes are expressed as partial eta-squared $\left(\eta_{\mathrm{p}}{ }^{2}\right)$ values.

There was a significant effect of cue type $[F(2,20)=$ $\left.38.973, p<.001, \eta_{\mathrm{p}}{ }^{2}=.796, p w=1.0\right]$, with reaction times to valid trials faster than those to neutral trials, which in turn were faster than those to invalid trials (see Table 1). A paired-samples $t$ test (two-tailed) between valid and invalid trials was significant $[t(10)=7.665, p<.001]$. These results cannot be explained by a speed/accuracy trade-off, since there was no significant difference in accuracy between the two conditions $[t(10)=1.15, p=.277]$. The trend was toward greater accuracy for responses to valid trials. The correct response to the tactile probe stimuli for all experiments was the release of the left pedal, regardless of the hand to which the stimulus was delivered.

Table 1 Effect of cue type on reaction times (RTs) to intermittent probe stimuli, along with percentages of errors (Exps. 1 and 2)

\begin{tabular}{lccll}
\hline \multicolumn{5}{c}{ Cue Type } \\
\cline { 2 - 5 } & Valid & Neutral & Invalid & Invalid - Valid \\
\hline Experiment & 1 & (Tactile Cues) & & \\
RT (ms) & 648 & 705 & 730 & $81(S D=35)$ \\
Errors (\%) & 0.5 & 1.4 & 1.8 & \\
Experiment 2 & (Visual Cues) & & $17(S D=24)$ \\
RT (ms) & 637 & 656 & 654 & \\
Errors (\%) & 2.5 & 2.5 & 3.3 &
\end{tabular}

Valid = cue and probe on same hand (Exp. 1)/in same location in external space (Exp. 2); Neutral = cues on both sides; Invalid = cue and probe on opposite hands (Exp. 1)/in opposite locations in external space (Exp. 2). 
The exogenous tap cues successfully manipulated spatial attention. Reaction times to tactile probes that had been cued were significantly faster (by $81 \mathrm{~ms}$ ) than reaction times to probes that had not been cued. This cuing effect is comparable to the 64-ms advantage for cued probes in Experiment 1 of Yates and Nicholls (2009).

\section{Prior entry effects}

For each participant, the proportion of "upper tap first" responses was plotted as a function of the SOA between the target taps for the three cuing conditions (lower tap cued, upper tap cued, or neutral-both taps cued). Cumulative normal distributions were fitted to the plotted responses; these fitted functions captured the participants' entire response distributions. The averaged data are shown in Fig. 4. The fitted functions were used to estimate the point of subjective simultaneity (PSS) for each of the three cuing conditions for each participant (derived as the SOA at which the fitted functions for the three cuing conditions crossed .5 on the $y$-axis-i.e., the point at which participants responded "upper tap first" and "lower tap first" equally often). Averaged PSS values are also presented in Fig. 4.

When the lower/thumb taps were cued (cue delivered to the same hand as the lower tap), the mean PSS was $+27 \mathrm{~ms}$, indicating that the PSS occurred when the upper tap preceded the lower tap by $27 \mathrm{~ms}$, implying that cuing the lower tap accelerated the perception of lower taps. The

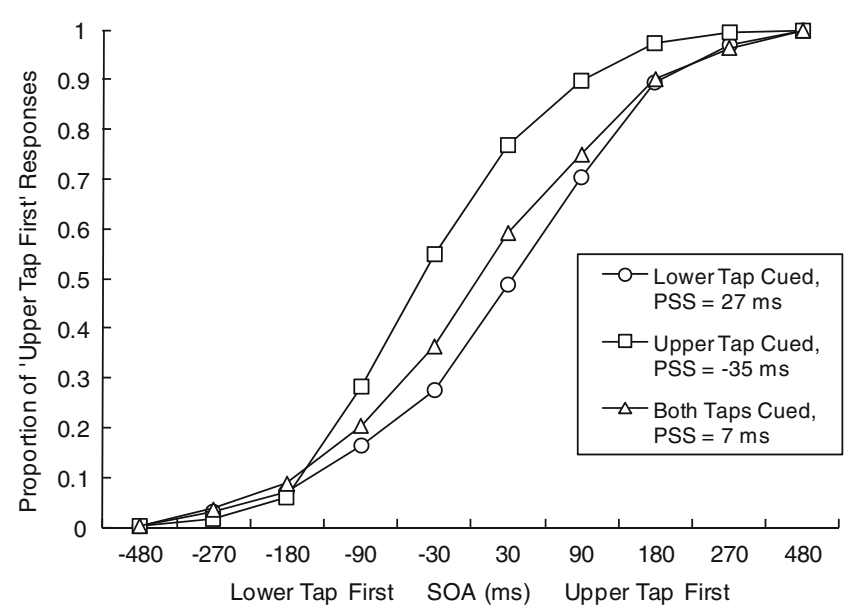

Fig. 4 Temporal order judgment data for Experiment 1 (tactile cuing): Average of the fitted data for all participants. Each step change in $\mathrm{SOA}$ is represented by an equal distance along the $x$-axis, (i.e., a nonratio axis, in the interest of magnifying the SOA region of most interest - the region close to true simultaneity). This was more relevant for later figures, but incorporated here in the interest of consistency across figures. PSS refers to mean points of subjective simultaneity. The "cued" tap is defined as the tactile target presented at the same external location as the tactile cue (i.e., the tactile target presented to the same hand as the tactile cue) mean PSS when the upper/index finger taps were cued was $-35 \mathrm{~ms}$ (i.e., when the lower tap preceded the upper tap by $35 \mathrm{~ms})$. The mean PSS when both taps were cued was +7 ms. PSS values were analyzed using a withinparticipants ANOVA with a single factor, Cue Condition, with three levels: lower tap cued, neutral (both taps cued), and upper tap cued.

There was a significant effect of cue condition $[F(2,20)=$ 7.503, $\left.p=.004, \eta_{\mathrm{p}}^{2}=.429, p w=.905\right]$ indicating that directing tactile attention to a particular location using exogenous cues affected the point at which participants judged two subsequent tap stimuli (one cued and one not cued) as simultaneous, as assessed using a TOJ task. The difference in the PSSs for the lower- and upper-tap-cued conditions was $62 \mathrm{~ms}$. A paired-samples $t$ test (two-tailed) conducted on the lower- and upper-tap-cued PSSs was significant $[t(10)=3.725, p=.004]$.

The magnitude of the prior-entry effect is calculated as the average shift in the PSS values across both the lowerand upper-tap-cued conditions, equivalent to half of the magnitude of the interval between the mean PSSs for the lower- and upper-tap-cued conditions (Shore et al., 2001). This yielded a prior-entry effect of $31 \mathrm{~ms}$, once again similar to Experiment 1 of Yates and Nicholls (2009), where it was calculated as $24 \mathrm{~ms}$. In the present experiment, the data were collapsed across task type (i.e., "which tap first?" and "which tap second?") prior to analysis. Values of PSSs derived in this way should theoretically be free of both first- and second-order response biases. Refer to Shore et al. (2001) for a discussion of this point.

This replication of the results of the earlier study lends further support to the existence of unimodal somatosensory prior entry when somatosensory exogenous cuing is employed. The exclusion in the present experiment of trials where gaze deviated from the central fixation cross allowed for a refinement of the original finding - the acceleration of perception can be attributed specifically to covert shifts of attention, not confounded by possible eye (or head) movements. The very small number of gaze deviations observed prior to exclusion $(<1 \%$ for this and all remaining experiments) indicates that participants' ability to comply with instructions to fixate centrally was good.

\section{Experiment 2}

The aim of the second experiment was to address the "sensory facilitation" account of perceptual acceleration of cued stimuli. The sensory facilitation account proposes that apparent prior-entry effects are related to local (peripheral) sensory interactions between cues and targets presented at nearby locations, rather than being a result of attention. This account is relevant only where exogenous cuing is 
used to manipulate attention, and is perhaps most persuasive as a potential explanation in the visual modality, where visual cues and visual targets have typically been presented at the same or similar retinotopic locations. In such circumstances, it is conceivable that presenting a cue at a particular location might lower response thresholds of neural populations coding for that location, such that targets presented there might be registered more rapidly, and hence might be perceived earlier, independent of any influence of attention (Schneider \& Bavelier, 2003). Yet it is also possible that sensory facilitation might be responsible for the apparent somatosensory/tactile prior-entry effects when tactile cues and targets are presented on the same hand, although it should be noted that cues and target stimuli were presented to different fingers of the same hand in the somatosensory prior-entry experiments. Sensory facilitation as an explanation of the results was considered for Experiment 1 of Yates and Nicholls (2009) but rejected on the grounds that the reaction time data from the intermittently presented probe stimuli were not consistent with a sensory facilitation account. If sensory facilitation rather than spatial attention shifts were responsible for the reaction time advantage for cued versus uncued probes, the reaction times to the validly cued probes should be no faster than those to neutral cued probes, because both involve the same degree of sensory facilitation for the cued probe. This was not the pattern of results observed in that experiment. Rather, reaction times to probes were significantly faster for validly cued probes than for neutral-cued probes, consistent with genuine spatial attention shifts. Accordingly, it was argued that sensory facilitation was not responsible for the perceptual acceleration observed in these experiments.

While this remains a valid point, a simpler and more direct means to rule out the sensory facilitation account is to present cues in a modality different from the modality of the target stimuli. This precludes any of the proposed within-modality interactions between cue and targets occurring at peripheral locations of the sensory epithelia (e.g., retina or hands). To this end, we replicated the design of Experiment 1, except that visual cues (brief flashes) were presented in place of the fifth-finger tactile cues employed in Experiment 1. These visual cues were presented on the left side, the right side, or both sides, at the same locations as the tactile targets. As with the tactile cues, the visual cues were spatially nonpredictive/uninformative and were designed to exogenously attract spatial attention.

Previous work has established that exogenous visual cues presented at lateral locations are capable of facilitating behavioral responses (faster and more accurate discrimination performance) to tactile targets presented in the vicinity of those visual cues (Kennett, Spence, \& Driver, 2002; Spence, Nicholls, Gillespie, \& Driver, 1998). What we sought to determine here was whether these nontactile (visual) cues could also accelerate the perceived timing of tactile targets at the cued location, thereby demonstrating that an acceleration of perception can be achieved under circumstances in which the influence of low-level sensory facilitation can be ruled out. Like Experiment 1, Experiment 2 employed a TOJ task to gauge the effects of cuing on the perceived timing of the tactile target stimuli.

\section{Method}

\section{Participants}

Sixteen healthy students took part in this experiment. The complete data sets (both reaction time and TOJ data) were excluded for 4 participants because the accuracy threshold of $70 \%$ (or greater) correct for the TOJs was not achieved. All of the remaining participants (8 female, 4 male) were strongly right-handed.

\section{Apparatus and stimuli}

The apparatus and stimuli for Experiment 2 were identical to those for Experiment 1 (refer to the Apparatus and Stimuli section for that experiment), except for the following: visual, rather than tactile, cues were used to manipulate spatial attention prior to the presentation of the two tactile target stimuli. These visual cues consisted of brief flashes of light presented on the left side, the right side, or both sides simultaneously. These cue flashes comprised a 20-ms activation of blue LED light(s) mounted on the left- and right-hand grips, located midway between the upper (index finger) tappers and lower (thumb) tappers of each hand (see Fig. 1).

Given that these visual cues replaced the tactile cues from Experiment 1, it was no longer necessary to secure vibrotactile stimulators to the fifth finger of each hand of participants in Experiment 2 (refer to Fig. 2). These tappers had delivered the tactile cues to manipulate spatial attention in Experiment 1.

The other classes of stimuli used in Experiment 2 (TOJ taps and reaction time probes) were identical to those used in Experiment 1.

\section{Procedure}

The procedure for Experiment 2 was identical to that for Experiment 1 (refer to the Procedure section for Exp. 1), except for the following: Visual, rather than tactile, cues were used to manipulate spatial attention prior to the presentation of the two tactile target stimuli.

Also, the onset of the first tactile target occurred $130 \mathrm{~ms}$ after cue offset (150 ms after the onset of the cue). The increased cue-target SOA was introduced because previous 
research (Exp. 1B of Spence et al., 2001) has shown that visual stimuli need to precede tactile stimuli by around $50 \mathrm{~ms}$ in order to be perceived as simultaneous. Consequently, visual cues should precede tactile targets by an additional $50 \mathrm{~ms}$ (relative to tactile cues) to maintain an equivalent subjective temporal separation between the cues and targets.

All other aspects of the procedure were identical to those of Experiment 1 (refer to the Procedure section of that experiment). Figure 3 illustrates the sequence of stimuli during a trial.

Results and discussion

\section{Cuing effects}

Mean reaction times to the intermittently presented tactile probe stimuli were analyzed as in Experiment 1. A validly cued tactile probe was one presented at the same location as the visual cue in external space. There was a nearsignificant effect of cue type $[F(2,22)=2.862, p=.079$, $\left.\eta_{\mathrm{p}}{ }^{2}=.206, p w=.503\right]$, with reaction times to valid trials faster than those to neutral and invalid trials, with invalid trials marginally faster than neutral trials (see Table 1). A paired-samples $t$ test (two-tailed) between valid and invalid trials was significant $[t(11)=2.421, p=.034]$. These results cannot be explained by a speed/accuracy trade-off, since there was no significant difference in accuracy between the two conditions $[t(11)=0.456, p=.658]$. The trend was toward greater accuracy for responses to valid trials.

In this experiment, where attention was exogenously manipulated by brief visual cues presented in the left or right hemispace, reaction times to cued tactile probes were faster than those to uncued tactile probes, although this advantage was more modest $(17 \mathrm{~ms})$ than that observed in earlier experiments in which both cues and probes were presented in the tactile modality.

To the best of our knowledge, this is, surprisingly, the first demonstration that spatially uninformative exogenous visual cues can facilitate tactile simple detection latencies for the cued tactile stimuli. Earlier studies that also examined the effects of visual cuing on tactile simple detection latencies did not demonstrate this specific finding. This was because they either employed spatially informative cuing - which would trigger endogenous shifts of attention (Butter, Buchtel, \& Santucci, 1989), thus obscuring the specific effect of exogenous shifts in spatial attention-or because they involved the presentation of tactile targets at a different location (on the shoulders) than the visual cues (on a computer screen in front of participants) (Tassinari \& Campara, 1996).

While our finding of facilitated tactile detection latencies at the visually cued location appears to be unique, it is nonetheless consistent with some related findings in the area. Indeed, we expected that the results from the reaction time probes employed here would largely mirror these related findings. In a covert spatial-orienting study, Spence et al. (1998) found that speeded tactile discrimination responses (continuous vs. pulsed vibration judgments) were faster and more accurate when spatially uninformative peripheral visual cues were presented on the same side as the tactile targets. Similarly, Kennett et al. (2002) also found faster and more accurate tactile discrimination responses (tactile elevation judgments) for tactile targets that had been cued by spatially uninformative exogenous visual cues. These findings from Spence et al. (1998) and Kennett et al. demonstrate that cuing in one modality (in this case, visual) can induce shifts in spatial attention in another modality (in this case, somatosensation). This is in line with other evidence showing that, for most modality pairings, altering the distribution of attention in one modality drives changes in the distribution of attention in another modality (Spence, McDonald, \& Driver, 2004). The reaction time data we observed in this experiment are consistent with this generally observed cross-modal cuing effect.

\section{Prior-entry effects}

Participants' responses were plotted as for Experiment 1, with cumulative normal functions fitted to the response distributions and PSS values for each of the three cuing conditions (lower tap cued, upper tap cued, or neutralboth taps cued) derived in the manner described earlier. The averaged data and PSS values are shown in Fig. 5.

When the lower/thumb taps were cued (visual cue delivered at the same location as the lower tap in external

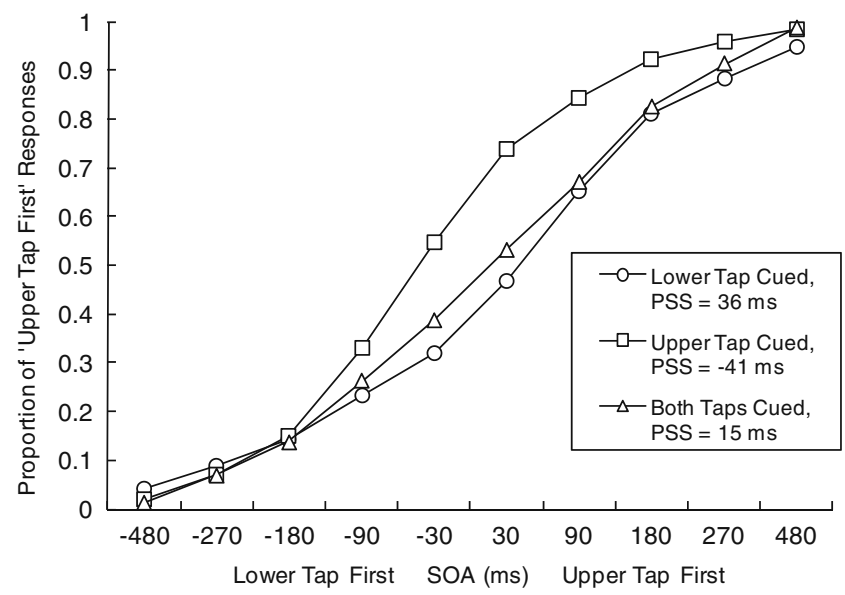

Fig. 5 Temporal order judgment data for Experiment 2 (visual cuing): Average of the fitted data for all participants. The "cued" tap is defined as the tactile target presented at the same external location as the visual cue 
space), the mean PSS was $+36 \mathrm{~ms}$, indicating that the PSS occurred when the upper tap preceded the lower tap by $36 \mathrm{~ms}$, implying that cuing the lower tap accelerated the perception of lower taps. The mean PSS when the upper/ index finger taps were cued was $-41 \mathrm{~ms}$ (i.e., when the lower tap preceded the upper tap by $41 \mathrm{~ms}$ ). The mean PSS when both taps were cued was +15 ms. PSS values were analyzed using a within-participants ANOVA with a single factor, Cue Condition, with three levels: lower tap cued, neutral (both taps cued), and upper tap cued.

There was a significant effect of cue condition $[F(2,22)=$ 12.641, $\left.p<.001, \eta_{\mathrm{p}}{ }^{2}=.535, p w=.991\right]$ indicating that directing visual attention to a particular location using exogenous cues affected the point at which participants judged two subsequent tactile stimuli (one at the same location as the visual cue and one on the opposite side) as simultaneous, as assessed using a TOJ task. The difference in the PSSs for the lower- and upper-tap-cued conditions was $77 \mathrm{~ms}$. A paired-samples $t$ test (two-tailed) conducted on the lower- and upper-tap-cued PSSs was significant $[t(11)=4.32$, $p=.001]$.

The TOJ data revealed that spatially attended target taps were perceived earlier than unattended target taps. The magnitude of this effect was $38 \mathrm{~ms}$, the largest magnitude observed to date for tactile prior entry. Importantly, the magnitude of tactile prior entry using visual cues was larger than when tactile cues were used, suggesting that the full prior-entry effect can be generated using cues in another modality, signifying not only that the effect can be generated under circumstances in which it is not possible for any low-level withinmodality sensory interactions to occur between cues and targets, but also that any influence of such sensory interactions in previous experiments that used tactile cues and targets would presumably be small, given that substituting tactile cues for visual cues does not diminish the magnitude of the prior-entry effect.

\section{Experiment 3}

The use of the TOJ task as a means of assessing the influence of attention on perceptual latencies has been criticized in the last decade, even allowing for the various methodological refinements that have been incorporated into more recent TOJ experiments specifically for the purpose of minimizing the influence of response bias (Shore \& Spence, 2005; Shore et al., 2001; Spence \& Parise, 2010). A growing number of researchers (Schneider \& Bavelier, 2003; Shore \& Spence, 2005; Zampini et al., 2005) have argued that an alternative method for gauging temporal perception, the SJ task, provides a superior measure, because it allows for an assessment of shifts in temporal perception that is genuinely free of response bias. This being the case, we tested for prior entry again, using the same cue and target permutations as in Experiments 1 and 2 (first with tactile cues, then with visual cues, and with tactile targets for both experiments), but this time using the SJ task. These were Experiments 3 and 4, respectively, which represent the first such investigations of tactile spatial prior entry using the SJ task.

The SJ task involves the presentation of two stimuli, which either are or are not simultaneous. Observers then report whether or not they perceive the stimuli as occurring simultaneously. In prior-entry studies that use this task, attention can be manipulated either exogenously or endogenously, such that attention is focused on one or the other target stimulus prior to their being presented. If attended stimuli are perceived earlier, as hypothesized, this should affect the SOA between the target stimuli at which observers maximally report them as simultaneous (i.e., the PSS). A complete account of the benefits of the SJ taskin particular, how it is an improvement on the TOJ task with respect to overcoming response bias-is provided by Schneider and Bavelier (2003).

Alongside the particular advantage of the SJ task in terms of overcoming response bias, another benefit of this approach is simply that it might provide a new, independent line of evidence in support of prior entry, adding greater weight to the original findings derived from the TOJ task.

These, then, are the general advantages of using the SJ as well as the TOJ task to measure the effect of attention on perceived timing, but there is also another specific benefit. The TOJ task used in Experiments 1 and 2 required participants to identify the order of two stimulus events. In these experiments, two tactile target stimuli were presented, one at an upper elevation and one at a lower elevation (on different hands). Given that participants had to judge whether the upper or the lower tap was presented first (or second), an obvious prerequisite for correct responding in this task was the ability to judge whether any individual tap was presented at the upper or the lower elevation, (i.e., the ability to compute the spatial location of the individual tactile targets, irrespective of their order). This means that the TOJ task requires both a temporal computation and a spatial computation. In contrast, in the SJ task, participants do not need to compute the location of the target stimuli in order to judge whether or not they occur simultaneously. For this reason, it is important when measuring the influence of any variable on temporal perception to compare the results from a TOJ task (which requires both temporal and spatial computations) with the results from an SJ task (which requires a temporal computation only). 
Method

\section{Participants}

Sixteen healthy students took part in this experiment. The complete data sets (both reaction time and SJ data) were excluded for 2 participants because of a failure to achieve the accuracy threshold, which was $60 \%$ (or greater) correct for the SJs. This accuracy threshold, which applied for both of our SJ task experiments, was less stringent than those for previous experiments that have used the TOJ task, reflecting the greater difficulty (and lower average accuracies) for the SJ task. All remaining participants ( 9 male, 5 female) were strongly right-handed.

\section{Apparatus and stimuli}

The apparatus and stimuli for Experiment 3 were identical to those for Experiment 1 (refer to the Apparatus and Stimuli section of Exp. 1), except for the following: $S J$ taps replaced the TOJ taps of the earlier experiment. The SJ taps consisted of brief (10-ms) vibrations of the two tappers secured to the index fingers of the two hands (i.e., one 10-ms vibration of the left-hand index finger tapper, and one $10-\mathrm{ms}$ vibration of the right-hand index finger tapper).

Given that these tactile SJ taps replaced the tactile TOJ taps used in Experiment 1, it was no longer necessary to secure vibrotactile stimulators to the thumb of each hand of participants in Experiment 3 (refer to Fig. 6). These thumb tappers were only necessary for the TOJ task.
The other classes of stimuli used in Experiment 3 (tactile cues and reaction time probes) were identical to those used in Experiment 1.

\section{Procedure}

The procedure for Experiment 3 was identical to that for Experiment 1 (refer to the Procedure section of that earlier experiment), except for the following: Tactile cues were followed by SJ trials (in five out of six trials) or reaction time probe trials (in one out of six trials), rather than TOJ trials (in five out of six trials) or reaction time probe trials (in one out of six trials). The SJ trials are described below.

SJ trials In five out of six trials, $80 \mathrm{~ms}$ after the offset of the cue (i.e., $100 \mathrm{~ms}$ after cue onset), the two SJ target taps were presented (one to each hand) with either synchronous onset ( $50 \%$ of the time) or asynchronous onset $(50 \%$ of the time). For the latter, the onsets were separated by one of 10 randomly assigned equiprobable intervals, ranging from -160 to $+160 \mathrm{~ms}(-160,-90,-60,-30,-10,+10,+30$, $+60,+90$, or $+160 \mathrm{~ms}$; positive values indicate that the right-hand tap was presented first). The equal probability of synchronous and asynchronous tap pairs eliminated the possibility that participants would select one response over the other on the basis of knowledge that one option occurred with greater frequency. Participants judged whether or not the two target taps delivered to the left and right hands were presented simultaneously and responded in a forced choice manner by releasing one of two pedals located under the toe and heel of the right foot. There was no requirement to
Fig. 6 Placement of stimuli for Experiments 3 and 4
Experiment 3
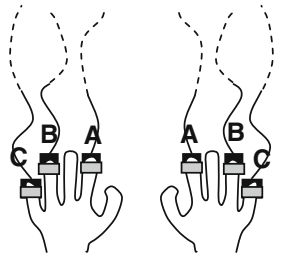

\begin{tabular}{|lll|}
\hline Location & Stimulus Type & Duration \\
\hline Index Finger (A) & Target Tap & $10 \mathrm{~ms}$ \\
Fourth Finger (B) & Reaction Time Probe & $200 \mathrm{~ms}$ \\
Fifth Finger (C) & Exogenous Cue Tap & $20 \mathrm{~ms}$ \\
\hline
\end{tabular}

Experiment 4

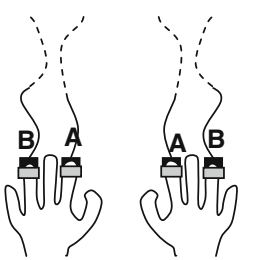

\begin{tabular}{|lll|}
\hline Location & Stimulus Type & Duration \\
\hline Index Finger (A) & Target Tap & $10 \mathrm{~ms}$ \\
Fourth Finger (B) & Reaction Time Probe & $200 \mathrm{~ms}$ \\
Secured to Hand Grip & Exogenous Cue Light & $20 \mathrm{~ms}$ \\
\hline
\end{tabular}


judge which tap (left or right) was presented first on those trials on which the taps were perceived to be presented asynchronously. Participants were divided into two groups. The first was instructed to release the toe pedal if they judged the onset of the taps as simultaneous, and the heel pedal if otherwise. The second group was given the converse instructions.

All other aspects of the procedure were identical to those in Experiment 1 (refer to Procedure section of that experiment). Figure 7 illustrates the sequence of stimuli during a trial.
Results and discussion

\section{Cuing effects}

Mean reaction times to the intermittently presented tactile probe stimuli were analyzed as in the previous experiments. There was a significant effect of cue type $[F(2,26)=$ $\left.19.127, p<.001, \eta_{\mathrm{p}}{ }^{2}=.595, p w=1.0\right]$, with reaction times to valid trials faster than those to neutral trials, which were in turn faster than those to invalid trials (see Table 2). A
Fig. 7 Sample sequence of events for a single trial in Experiments 3 and 4 . Cuing was either tactile (Exp. 3) or visual (Exp. 4). The experimental stimuli were identical to those for Experiments 3 and 4. The central fixation cross was displayed until a response was made

\section{Stage 1 - Cuing}

Experiment 3 (Tactile Cuing)

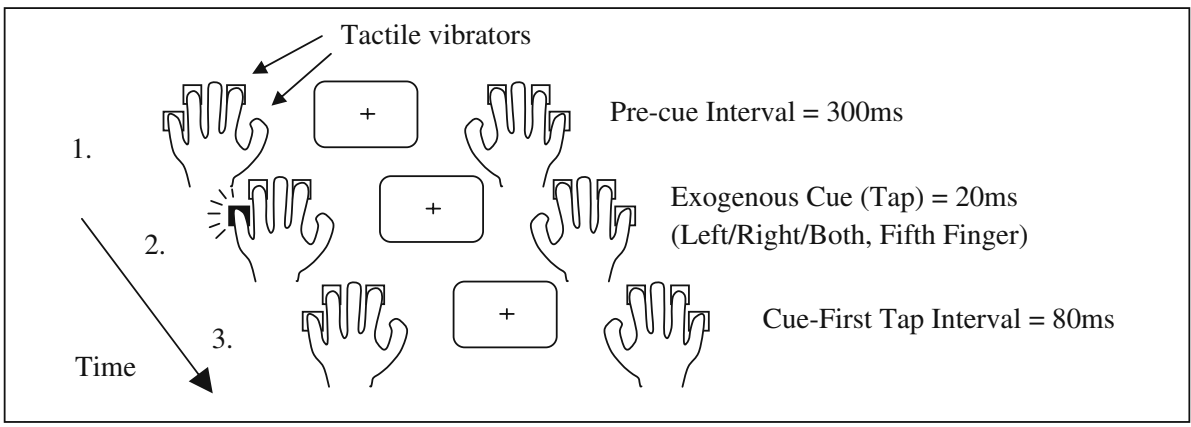

Experiment 4 (Visual Cuing)

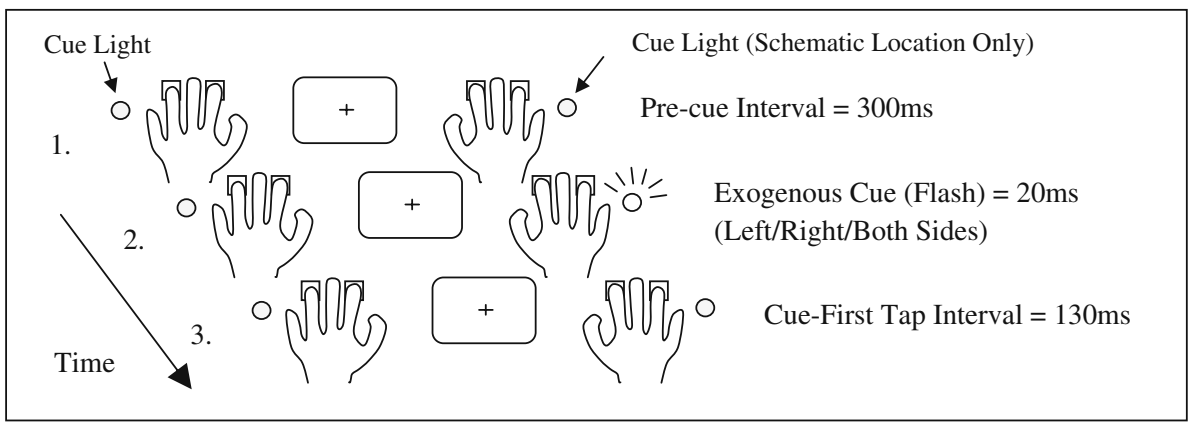

Stage 2 - Experimental Stimuli (Experiments 3 and 4)

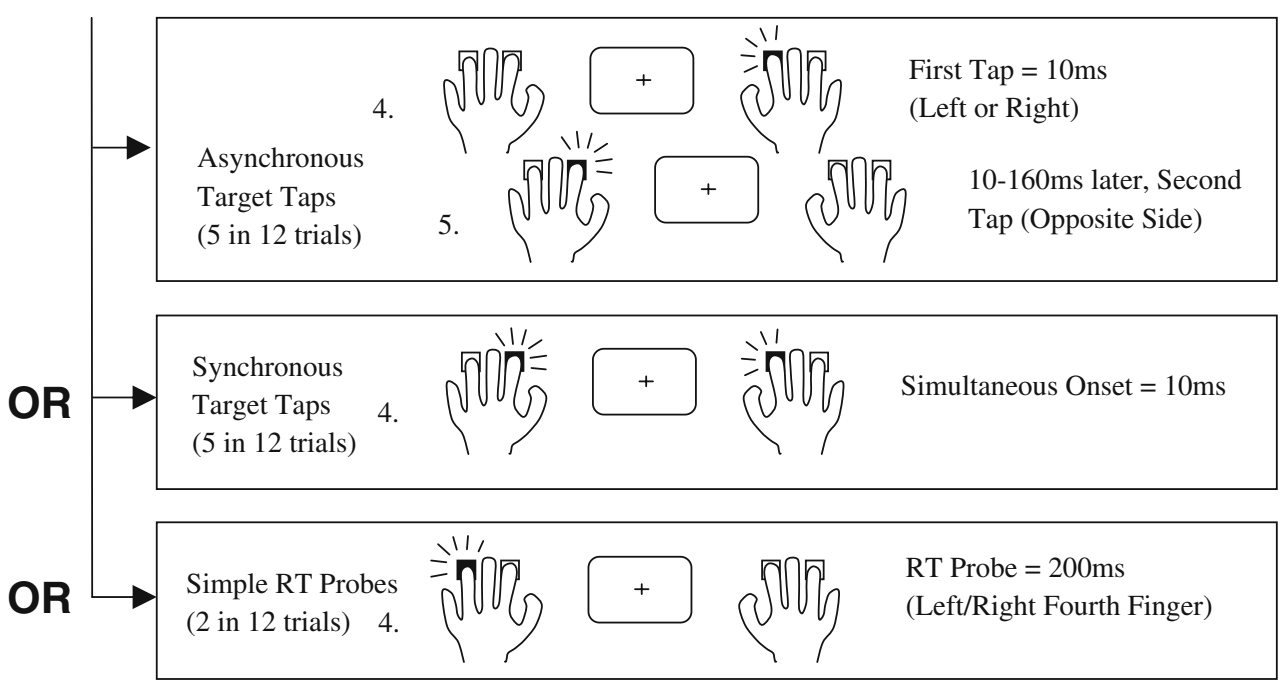


Table 2 Effect of cue type on reaction times (RTs) to intermittent probe stimuli, along with percentages of errors (Exps. 3 and 4)

\begin{tabular}{|c|c|c|c|c|}
\hline & \multicolumn{4}{|c|}{ Cue Type } \\
\hline & Valid & Neutral & Invalid & Invalid - Valid \\
\hline \multicolumn{5}{|c|}{ Experiment 3 (Tactile Cues) } \\
\hline RT (ms) & 615 & 648 & 664 & $49(S D=34)$ \\
\hline Errors $(\%)$ & 1.8 & 1.1 & 4.6 & \\
\hline \multicolumn{5}{|c|}{ Experiment 4 (Visual Cues) } \\
\hline RT (ms) & 615 & 654 & 633 & $19(S D=28)$ \\
\hline Errors $(\%)$ & .7 & 6.3 & 5.0 & \\
\hline
\end{tabular}

Valid $=$ cue and probe on same hand (Exp. 3)/in same location in external space (Exp. 4); Neutral = cues on both sides; Invalid = cue and probe on opposite hands (Exp. 3)/in opposite locations in external space (Exp. 4).

paired-samples $t$ test (two-tailed) between valid and invalid trials was significant $[t(13)=5.388, p<.001]$. These results cannot be explained by a speed/accuracy trade-off, since there was no significant difference in accuracy between the two conditions $[t(13)=1.529, p=.15]$. The trend was toward greater accuracy for responses to valid trials.

The effect of cuing on reaction times to tactile probes was comparable to that observed in Experiment 1. This was anticipated, given that the reaction time probe task was identical in the two experiments. Spatial attention was successfully manipulated by the exogenous tap cues, with reaction times to cued probes significantly faster-by $49 \mathrm{~ms}$ - than those to uncued probes. By comparison, in Experiment 1, participants' responses to cued versus uncued probes were significantly faster, by $81 \mathrm{~ms}$.

\section{Prior entry effects}

For each participant, the proportion of "simultaneous" responses was plotted as a function of the SOA between the onset of the target taps for the three cuing conditions (left hand cued, right hand cued, or both hands simultaneously cued). The observed data for each participant for each of the three conditions were fitted to a Gaussian function using maximum likelihood estimation (see Myung, 2003). The averaged data are shown in Fig. 8. The means of these functions were used to estimate the PSSs for each of the three cuing conditions; the averaged PSS values are also presented in Fig. 8.

The mean PSS for the left-hand-cued condition was $+6 \mathrm{~ms}$ (indicating that the PSS occurred when the right-hand target tap preceded the left-hand target tap by $6 \mathrm{~ms}$ ). The mean PSS for the right-hand-cued condition was $-6 \mathrm{~ms}$. The mean PSS when both hands were cued was $-3 \mathrm{~ms}$. These left- and righthand-cued mean PSS values are in the direction predicted by the prior-entry hypothesis. PSS values were analyzed using a within-participants ANOVA with a single factor, Cue Condition, with three levels: left hand cued, neutral (both hands cued), or right hand cued.

There was a significant effect of cue condition $[F(2,26)=$ 3.493, $\left.p=.045, \eta_{\mathrm{p}}{ }^{2}=.212, p w=.6\right]$, indicating that directing tactile attention to a particular location using exogenous cuing affected the point at which participants judged two subsequent tap stimuli (one cued and one not cued) as simultaneous, as assessed using an SJ task. The difference in the PSSs for the left- and the right-hand-cued conditions was $12 \mathrm{~ms}$. A paired-samples $t$ test (two-tailed) conducted on the left- and right-hand-cued PSS values was significant $[t(13)=3.448, p=.004]$.

The magnitude of the prior-entry effect in this experiment (the average amount of the shift from actual simultaneity across the left- and right-hand-cued conditions) was $6 \mathrm{~ms}$. As with Experiment 1, the exclusion of trials on which gaze deviated from the central fixation cross meant that the observed acceleration of perception can be attributed to covert shifts of attention, not confounded by possible eye (or head) movements.

The establishment of prior entry using an SJ task reinforces the earlier finding of prior entry using a TOJ task, indicating that this is a robust effect. However, the magnitude of the prior-entry effect for the SJ task, $6 \mathrm{~ms}$, was considerably smaller than that obtained for the TOJ task in Experiment 1 of the present study-31 ms-as well as for Experiment 1 of Yates and Nicholls (2009) where it was $24 \mathrm{~ms}$. The latter estimates were arrived at after the influence of response bias had supposedly been accounted for. While some disparity between the priorentry estimates for the different tasks might be expected,

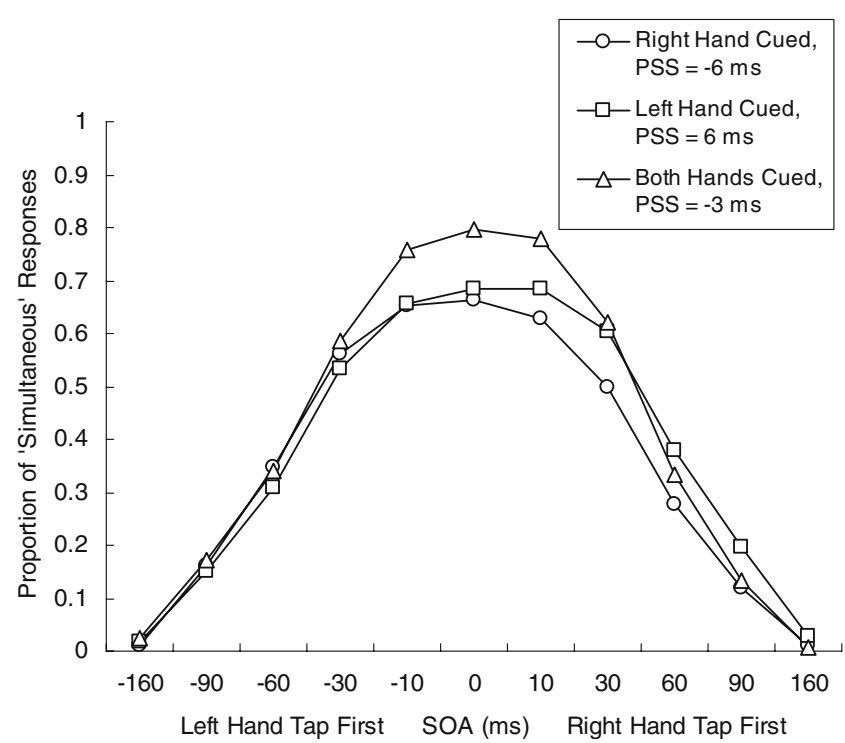

Fig. 8 Simultaneity judgment data for Experiment 3 (tactile cuing): Average of the fitted data for all participants 
given that the PSS values for each were derived via different methods (e.g., the range of SOAs separating the experimental stimuli was narrower for the SJ task), this account alone does not appear sufficient to explain the discrepancy. Nor can the disparity be readily attributed to changes in the effectiveness of the cues in manipulating spatial attention across the different experiments, since these were generally comparable (TOJ task experiments, 64 and $81 \mathrm{~ms}$, respectively; the present SJ task experiment, $49 \mathrm{~ms}$ ). This therefore suggests that either response bias was not truly eliminated in the TOJ tasks (even with designs that featured orthogonal cuing and response dimensions as well as averaging across "which stimulus first" and "which stimulus second" instruction manipulations) or, potentially, that the TOJ and SJ tasks measure different aspects of temporal perception (see van Eijk et al., 2008, for a discussion of this interesting possibility, and refer to the General Discussion below for additional possible accounts of the TOJ/SJ disparity). Importantly, progress in understanding how subjective time is represented in the brain will be limited until the discrepancy between SJ and TOJ task measures of perceived timing is satisfactorily clarified.

\section{Experiment 4}

Experiment 4 was a replication of Experiment 2 (visual cues and tactile targets) using an SJ task in place of the TOJ task (and can alternatively be described as a replication of Exp. 3 using visual cues in place of tactile cues). If the prior-entry effect found in Experiment 2, which used visual cues, is replicated with the SJ task, this would represent the most convincing evidence yet of prior entry, since both the sensory facilitation and response bias accounts of the results could be ruled out.

Method

\section{Participants}

Twenty healthy students took part in this experiment. The complete data set (both reaction time and SJ data) was excluded for 1 participant because of a technical failure of the equipment during the experiment (malfunction of the headphones). All of the remaining participants (13 female, 6 male) were strongly right-handed.

\section{Apparatus and stimuli}

The apparatus and stimuli for Experiment 4 were identical to those in Experiment 3 (refer to the Apparatus and Stimuli section for that experiment), except for the following: visual, rather than tactile, cues were used to manipulate spatial attention prior to the presentation of the two tactile target stimuli. These visual cues consisted of brief flashes of light presented on the left side, the right side, or both sides simultaneously. These cue flashes comprised a 20 -ms activation of blue LED light(s) mounted on the left and right hand grips, located below the index finger tappers of the two hands (see Fig. 1).

Given that these visual cues replaced the tactile cues used in Experiment 3, it was no longer necessary to secure vibrotactile stimulators to the fifth finger of each hand of participants in Experiment 3 (refer to Fig. 6). These tappers delivered the tactile cues to manipulate spatial attention in Experiment 3.

The other classes of stimuli used in Experiment 4 (simultaneity judgment taps and reaction time probes) were identical to those used in Experiment 3.

\section{Procedure}

The procedure for Experiment 4 was identical to that of Experiment 3 (refer to the Procedure section for that experiment), except for the following: visual, rather than tactile, cues were used to manipulate spatial attention prior to the presentation of the two tactile target stimuli.

Also, the onset of the first tactile target occurred $130 \mathrm{~ms}$ after cue offset (150 ms after the onset of the cue) in order to maintain a subjective temporal separation between cues and targets equivalent to that in Experiment 3 (refer to the Procedure section of Exp. 2 for the rationale of this cue-target SOA).

All other aspects of the procedure were identical to those in Experiment 3. Figure 7 illustrates the sequence of stimuli during a trial.

\section{Results and discussion}

Cuing effects

Mean reaction times to the intermittently presented tactile probe stimuli were analyzed as previously via a withinparticipants ANOVA with a single factor, Cue Type, with three levels: valid, neutral, and invalid. A validly cued tactile probe was one presented at the same location in external space as the visual cue.

There was a significant effect of cue type $[F(2,36)=$ 14.401, $\left.p<.001, \eta_{\mathrm{p}}{ }^{2}=.444, p w=.998\right]$, with reaction times to valid trials faster than those to both neutral and invalid trials, although neutral trials were faster than invalid trials (see Table 2). A paired-samples $t$ test (two-tailed) between valid and invalid trials was significant $[t(18)=$ 2.894, $p=.01]$. These results cannot be explained by a speed/accuracy trade-off, since there was no significant 
difference in accuracy between the two conditions $[t(18)=$ $1.045, p=.310]$. The trend was toward greater accuracy for responses to valid trials.

As anticipated, and consistent with the TOJ-task-based Experiment 2 that this experiment replicates (except that, here, the SJ task was used), reaction times to visually cued tactile probes were faster than those to uncued tactile probes. The reaction time advantage $(19 \mathrm{~ms})$ was almost identical to that observed in Experiment 2 (17 ms), suggesting that this is a robust phenomenon. An unexpected finding was that responses to tactile probes were slowest in the neutral condition, in which visual cues were presented on both sides. An echo of this result was observed in Experiment 2, although in that case the tactile probe reaction times were only marginally slower in the neutral condition, not significantly so. A possible account for the result in the present experiment is that the neutral condition (two flashes) was less expected than the lateral cuing conditions (which both involved only a single flash), given that it occurred on only one in three trials, as compared to the single-flash conditions, which occurred on two in three trials. This reduced probability of receiving a double flash (neutral condition) relative to a single flash (in both lateral conditions) may have retarded reaction times to tactile probes in the neutral condition.

\section{Prior entry effects}

Participants' responses were plotted as for Experiment 3. The averaged data and PSS values are shown in Fig. 9.

The mean PSS for the left-hand-cued condition was $+6 \mathrm{~ms}$ (indicating that the PSS occurred when the right-hand target tap preceded the left-hand target tap by $6 \mathrm{~ms}$ ). The mean PSS for the right-hand-cued condition was $-7 \mathrm{~ms}$. The mean PSS for the both-hands-cued condition was $0 \mathrm{~ms}$. The left- and right-hand-cued mean PSS values are in the direction predicted by the prior-entry hypothesis. The PSS values were analyzed using a within-participants ANOVA with a single factor, Cue Condition, with three levels: left cue, neutral (both cues), or right cue.

There was a near-significant effect of cue condition $\left[F(2,36)=3.107, p=.057, \eta_{\mathrm{p}}{ }^{2}=.147, p w=.562\right]$, suggesting that directing visual attention to a particular location using exogenous cuing (brief flashes) affected the point at which participants judged two subsequent tap stimuli (one cued and one not cued) as simultaneous, as assessed using an SJ task. The difference in the PSSs for the left- and right-hand-cued conditions was (rounded to) $12 \mathrm{~ms}$. A paired-samples $t$ test (two-tailed) conducted on the left- and right-hand-cued PSS values was significant $[t(18)=2.683, p=.015]$.

The data for prior entry revealed that spatially attended target taps were perceived earlier than unattended target taps (the valid vs. invalid cuing $t$ test was significant, and

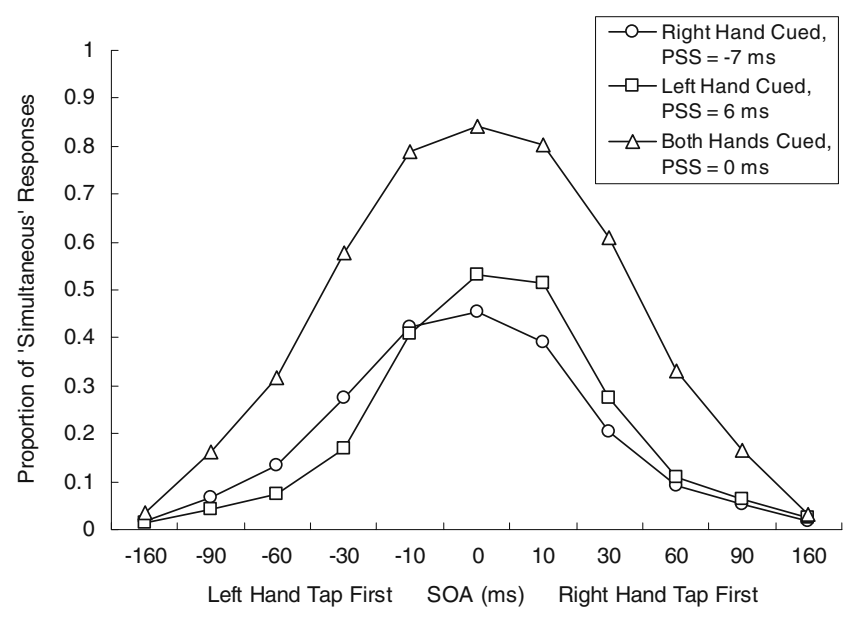

Fig. 9 Simultaneity judgment data for Experiment 4 (visual cuing): Average of the fitted data for all participants

an $F$ test of all cuing conditions was just short of significance). The magnitude of this effect was $6 \mathrm{~ms}$, smaller than the magnitude derived from the TOJ task (38 ms), in keeping with a number of results showing that the magnitude of time perception shifts is generally smaller when measured using the SJ task than using the TOJ task (cf. Exps. 1 and 3 of this study; Schneider \& Bavelier, 2003; Schwarz \& Eiselt, 2009; Shore et al., 2001; but see Vatakis, Navarra, Soto-Faraco, \& Spence, 2008). Notably, this is arguably the most compelling demonstration of an exogenously generated spatial prior-entry effect yet produced, given that here the sensory facilitation, overt orienting, and response bias confounds can be decisively rejected as competing accounts to genuine prior entry.

\section{General discussion}

Experiment 1 of this study replicated Experiment 1 of Yates and Nicholls (2009), though with some improvements to the experimental design, most notably the introduction of eye movement monitoring. Both experiments investigated somatosensory prior entry with spatially uninformative exogenous tactile cuing, and both found a modest priorentry effect, around 25-30 ms. An advantage of the present Experiment 1 over the original study is that this effect could be attributed more convincingly to shifts in covert spatial attention, given that eye movements were monitored and trials with deviations of gaze from the central fixation point were excluded in the present study.

Experiment 2 was identical to Experiment 1, except that visual cues replaced the tactile cues. The use of visual rather than tactile cues circumvented the concern that the earlier perception of attended stimuli might be attributable to local sensory interactions between cues and targets, 
rather than to an effect of spatial attention per se. Despite the substitution of visual for tactile cues, a prior-entry effect was observed in Experiment 2, this time of nearly $40 \mathrm{~ms}$, indicating that spatial attention rather than low-level sensory facilitation was responsible for this particular deviation from veridical time perception.

Experiments 3 and 4 were conducted with the same pairings of cues and targets as in Experiments 1 and 2, respectively (i.e., in Exp. 3, tactile cues, tactile targets; in Exp. 4, visual cues, tactile targets). With Experiments 3 and 4, we sought to establish whether the results from Experiments 1 and 2 would be replicated when an entirely different method for assessing perceived timing was employed, the SJ task. The rationale for this was threefold. First, the existence of two independent sources of evidence converging on the same conclusion provides a more compelling existence proof of prior entry than either one alone. Second, the new method-incorporating the SJ task-more convincingly eliminates the lingering response bias confounds associated with the TOJ task used in Experiments 1 and 2. Finally, the SJ task differs from the TOJ task in that it does not require an explicit spatial computation, thereby removing this potential confound. In both Experiments 3 and 4, prior-entry effects were observed, although the magnitude of the effect $-6 \mathrm{~ms}$ for both experiments - was much smaller than when assessed with the TOJ task. These results represent the first demonstration of somatosensory prior entry using the SJ task. The equivalent magnitudes of the effect, regardless of whether tactile or visual cues were employed, strengthen the argument that local cue-target sensory interactions are not behind these shifts in perceived timing.

With the addition of this study, the hypothesis of spatial prior entry has now been tested, using both TOJ and SJ tasks, for the visual, auditory, and tactile modalities. In studies using the TOJ task, spatial prior entry has been demonstrated for all of these modalities, at least when spatial attention was manipulated exogenously. Visual spatial prior entry has been demonstrated (with the TOJ task) by Shore et al. (2001), Hongoh, Kita, and Soeta (2008), McDonald, Teder-Sälejärvi, Di Russo, and Hillyard (2005), and Santangelo and Spence (2009). Auditory spatial prior entry has been demonstrated using the TOJ task by Spence and Lupiáñez (1998). Tactile spatial prior entry has been demonstrated using the TOJ task by van Damme, Gallace, Spence, Crombez, and Moseley (2009), Yates and Nicholls (2009), and Experiments 1 and 2 of the present study. Spatial prior entry using endogenous manipulations of spatial attention has been demonstrated (using the TOJ task) for the visual (Shore et al., 2001) and tactile (Yates \& Nicholls, 2009) modalities only. In the exogenous cuing studies in all modalities, spatial prior entry has been demonstrated in experiments that employed cues in modalities different from those of the target stimuli, thereby precluding intramodal sensory facilitation as an explanation for the observed effects (e.g., visual spatial prior entry with auditory cues-Hongoh et al., 2008; McDonald et al., 2005; Santangelo \& Spence, 2009; auditory spatial prior entry with visual cues-Spence \& Lupiáñez, 1998; tactile spatial prior entry with visual cues-van Damme et al., 2009, and Exp. 2 of the present study). Most of the TOJ studies described above, whether they used exogenous or endogenous cuing, employed orthogonal designs, thereby eliminating the potential confound of simple response bias.

Clear demonstrations of spatial prior entry using the SJ task are less common, relative to the TOJ task. We are aware of only two examples - the present study, which established spatial prior entry for tactile stimuli using both tactile and visual spatial cuing, and the study of Santangelo and Spence (2008), which established spatial prior entry for visual stimuli with auditory spatial cuing. While Schneider and Bavelier (2003) found a similar effect using an SJ task (except with visual spatial cuing), they concluded, based on subsequent experiments, that the effect was likely attributable to sensory facilitation. Kanai et al. (2007) found no evidence of spatial prior entry with auditory stimuli when using an SJ task.

Differences between magnitude estimates of prior entry using TOJ and SJ tasks

A central feature of our findings was the consistent difference in the magnitudes of prior entry derived from the SJ and the TOJ task. It is important to be cautious in interpreting this difference, given the differences across the two tasks in terms of both the presentation of the experimental stimuli and the manner in which the PSS values were derived. Nevertheless, the TOJ/SJ magnitude dissociation observed in the present experiments is emerging as a consistent feature of investigations into prior entry (Kanai et al., 2007; Schneider \& Bavelier, 2003; Shore et al., 2001; Stelmach \& Herdman, 1991; van der Burg, Olivers, Bronkhorst, \& Theeuwes, 2008; Zhuang \& Papathomas, 2009; as well as both pairs of TOJ/SJ task experiments in the present study) and deserves consideration. The existence of a TOJ/SJ magnitude difference is also relevant to a central question in neuroscience, which is how the timing of brain activity relates to the timing of perception. An interesting study by McDonald et al. (2005) demonstrated (using a TOJ task) that auditory cuing significantly accelerated the perception of visual stimuli, yet had "little or no effect on the peak latencies of the principal [visual] ERP components" (p. 1199). It was argued that this "provides clear evidence that the timing of visual perception is not inevitably based on the timing of neural events in the visual-cortical pathways" (p. 1201). A 
different conclusion might have been reached, however, if the study had assessed the effect of auditory cuing on the perceived timing of visual stimuli using an SJ task. In that event, it seems likely that the effect would have been estimated as much smaller in magnitude, and indeed broadly comparable in magnitude to the effect of cuing on the timing of neural events. Such a finding would be consistent with the hypothesis that the timing of visual perception is based on the timing of neural events in the visual-cortical pathways (see also Nikolov, Rahnev, \& Lau, 2010, for a discussion of the findings of McDonald et al.'s, 2005, study).

Why, though, might there be a difference between the magnitude of prior entry as measured with a TOJ task and an SJ task? Conceivably, participants' prior knowledge of the type of judgment they are to make concerning the stimuli (i.e., SJ or TOJ) before the stimuli are presented might play a role. This could be tested by presenting the stimuli first, and then only afterward instructing participants on the type of judgment to perform. Another possibility is that, at any given SOA between the target stimuli, participants are generally more certain of whether or not the stimuli are simultaneous, relative to the order in which they are presented. These variations in (un)certainty might affect the degree to which attention influences temporal perception (i.e., the influence of attention on temporal perception may be reduced under conditions of greater certainty about the correct response). Other investigators (Shore \& Spence, 2005; van Eijk et al., 2008) have proposed that the SJ task is simply a less sensitive measure of changes in temporal perception than is the TOJ task, though exactly why this should be is unclear. An alternative interpretation is that differences in PSS values between SJ and TOJ tasks reflect "differences in the underlying perceptions that are being measured" (van Eijk et al., 2008, p. 964) as opposed to differences in the sensitivity of the two measures to the same underlying perceptual phenomenon. van Eijk et al. supported this conclusion on the basis of a lack of correlation between the SJ and TOJ PSS values in their study of audiovisual synchrony. A further possibility is that the nontemporal processing typically required in TOJ tasks in addition to the temporal processing may underlie the differences in PSS values between SJ and TOJ tasks (e.g., the TOJ experiments of the present study required elevation discriminations, orientation discriminations were required by Shore et al., 2001, and color discriminations by McDonald et al., 2005). Finally, as considered earlier, the difference might occur because PSS values derived from TOJ tasks are exaggerated due to response biases, despite the efforts to minimize these biases. At present, there are too few data to distinguish between these various alternatives.
Cross-modal "temporal ventriloquism" does not account for the earlier perception of visually cued tactile stimuli

Experiments 2 and 4, both of which incorporated visual cues and tactile targets, were specifically designed to overcome concerns about intramodal sensory facilitation between cues and targets as a potential non-attention-based explanation of the earlier perception of cued targets. These experiments clearly demonstrated that perception of cued targets is accelerated in the absence of any possible intramodal sensory facilitation. Yet other forms of crossmodal sensory facilitation have been proposed. One such account, less frequently considered in the literature than intramodal sensory facilitation - at least in relation to prior entry - is that of "temporal ventriloquism" (see, e.g., Schneider \& Bavelier, 2003). This is the possibility that cue and target stimuli, even if they are presented in different sensory modalities, are temporally attracted to each other (i.e., are perceived closer together in time than their actual temporal separation), provided that they are separated by only a short time interval (Bertelson \& Aschersleben, 2003; Morein-Zamir, Soto-Faraco, \& Kingstone, 2003). As an explanation of apparent prior-entry effects, the argument is that if this temporal ventriloquism effect were in operation, the cued target stimulus would be perceived to occur earlier than the uncued target stimulus because of a shift in its perceived temporal location toward the cue that preceded it, not because of attention. However, recent work by Keetels and Vroomen (2008), which specifically investigated temporal ventriloquism between tactile and visual stimuli (the modality pairing used for Exps. 2 and 4), established that the effect is not influenced by the spatial separation of the stimuli. That is, "the stimuli in one sensory modality [in this case, the visual cues] have just as much effect on the temporal perception of stimuli presented in the other modality [the tactile target stimuli] regardless of whether they are presented from the same versus from different locations" (Spence \& Parise, 2010, p. 372). These authors continued, to make an important point: "Hence, it turns out that the phenomenon of temporal ventriloquism simply cannot be used to explain the spatially-specific cuing effects seen in cross-modal exogenous cuing studies of the priorentry effect" (p. 372). While temporal ventriloquism can be ruled out, it may yet be demonstrated that the earlier perception of cued target stimuli is nevertheless the result of some other variant of cross-modal sensory facilitation (see Arabzadeh, Clifford, \& Harris, 2008; Harris, Arabzadeh, Moore, \& Clifford, 2007; Rowland, Quessy, Stanford, \& Stein, 2007). This remains a notable research question, but if such an effect were in operation, it would be expected to facilitate reaction times to validly and neutrally cued tactile probes equally. This is difficult to reconcile with the pattern of results observed in Experiments 2 and 4, again 
suggesting that attention is genuinely responsible for the observed earlier perception of cued tactile targets.

Future research directions

A welcome addition to the findings in this study would be measures of neural activity that correspond to the observed perceptual and behavioral phenomena described here. High-temporal-resolution somatosensory evoked potential (SEP) studies of spatial prior entry will likely provide the dominant source of data in this regard in the near future, although this line of evidence is limited by the fact that each component of the evoked potential is a summation of activity "from large groups of neurons that may have different latencies" (Lee, Williford, \& Maunsell, 2007, p. 9636) and by uncertainties about the spatial origin of the neural activity that underlies SEP components. Ultimately, single-unit studies offer greater scope to uncover the neural basis of spatial prior entry.

Author Note The authors thank Max Rademacher for technical assistance. We gratefully acknowledge the support of Australian Research Council Grant DP0557576 to M.E.R.N.

\section{References}

Allan, L. G. (1975). The relationship between judgments of successivenss and judgments of order. Perception \& Psychophysics, 18, 29-36.

Arabzadeh, E., Clifford, C. W. G., \& Harris, J. A. (2008). Vision merges with touch in a purely tactile discrimination. Psychological Science, 19, 635-641.

Bertelson, P., \& Aschersleben, G. (2003). Temporal ventriloquism: Crossmodal interaction on the time dimension-1. Evidence from auditory-visual temporal order judgment. International Journal of Psychophysiology, 50, 147-155.

Butter, C. M., Buchtel, H. A., \& Santucci, R. (1989). Spatial attentional shifts - Further evidence for the role of polysensory mechanisms using visual and tactile stimuli. Neuropsychologia, 27, 1231-1240.

Cairney, P. T. (1975). Bisensory order judgment and prior entry hypothesis. Acta Psychologica, 39, 329-340.

Coull, J. T., \& Nobre, A. C. (1998). Where and when to pay attention: The neural systems for directing attention to spatial locations and to time intervals as revealed by both PET and fMRI. The Journal of Neuroscience, 18, 7426-7435.

Dennett, D. C., \& Kinsbourne, M. (1992). Time and the observerThe where and when of consciousness in the brain. The Behavioral and Brain Sciences, 15, 183-201.

Eagleman, D. M., Tse, P. U., Buonomano, D., Janssen, P., Nobre, A. C., \& Holcombe, A. O. (2005). Time and the brain: How subjective time relates to neural time. The Journal of Neuroscience, $25,10369-10371$.

Harris, J. A., Arabzadeh, E., Moore, C. A., \& Clifford, C. W. G. (2007). Noninformative vision causes adaptive changes in tactile sensitivity. The Journal of Neuroscience, 27, 7136-7140.

Hongoh, Y., Kita, S., \& Soeta, Y. (2008). Separation between sound and light enhances audio-visual prior entry effect. IEICE Transactions on Information and Systems, E91.D, 1641-1648.
Jaśkowski, P. (1991). Two-stage model for order discrimination. Perception \& Psychophysics, 50, 76-82.

Kanai, K., Ikeda, K., \& Tayama, T. (2007). The effect of exogenous spatial attention on auditory information processing. Psychological Research, 71, 418-426.

Keetels, M., \& Vroomen, J. (2008). Tactile-visual temporal ventriloquism: No effect of spatial disparity. Perception \& Psychophysics, 70, 765-771.

Kennett, S., Spence, C., \& Driver, J. (2002). Visuo-tactile links in covert exogenous spatial attention remap across changes in unseen hand posture. Perception \& Psychophysics, 64, 1083-1094.

Köhler, W. (1947). Gestalt psychology: An introduction to new concepts in modern psychology. New York: Liveright.

Lee, J., Williford, T., \& Maunsell, J. H. R. (2007). Spatial attention and the latency of neuronal responses in macaque area v4. The Journal of Neuroscience, 27, 9632-9637.

McDonald, J. J., Teder-Sälejärvi, W. A., Di Russo, F., \& Hillyard, S. A. (2005). Neural basis of auditory-induced shifts in visual timeorder perception. Nature Neuroscience, 8, 1197-1202.

Morein-Zamir, S., Soto-Faraco, S., \& Kingstone, A. (2003). Auditory capture of vision: Examining temporal ventriloquism. Cognitive Brain Research, 17, 154-163.

Myung, I. J. (2003). Tutorial on maximum likelihood estimation. Journal of Mathematical Psychology, 47, 90-100.

Nikolov, S., Rahnev, D. A., \& Lau, H. (2010). Probabilistic model of onset detection explains paradoxes in human time perception. Frontiers in Consciousness Research, 1, 37.

Oldfield, R. C. (1971). Assessment and analysis of handedness: The Edinburgh inventory. Neuropsychologia, 9, 97-113.

Rowland, B. A., Quessy, S., Stanford, T. R., \& Stein, B. E. (2007). Multisensory integration shortens physiological response latencies. The Journal of Neuroscience, 27, 5879-5884.

Santangelo, V., \& Spence, C. (2008). Crossmodal attentional capture in an unspeeded simultaneity judgement task. Visual Cognition, $16,155-165$.

Santangelo, V., \& Spence, C. (2009). Crossmodal exogenous orienting improves the accuracy of genuinely-unspeeded temporal order judgments. Experimental Brain Research, 194, 577-586.

Schneider, K. A., \& Bavelier, D. (2003). Components of visual prior entry. Cognitive Psychology, 47, 333-366.

Schwarz, W., \& Eiselt, A. K. (2009). The perception of temporal order along the mental number line. Journal of Experimental Psychology. Human Perception and Performance, 35, 989-1004.

Shore, D., \& Spence, C. (2005). Prior entry. In L. Itti, G. Rees, \& J. Tsotsos (Eds.), Neurobiology of attention (pp. 89-95). Amsterdam: Elsevier.

Shore, D., Spence, C., \& Klein, R. M. (2001). Visual prior entry. Psychological Science, 12, 205-212.

Spence, C., \& Lupiáñez, J. (1998). Crossmodal links in attention revealed by the orthogonal temporal order judgment task. Paper presented at the II Congreso de la Sociedad Espanyola de Psicologica Experimental (SEPEX '98), Granada, Spain.

Spence, C., McDonald, J., \& Driver, J. (2004). Exogenous spatialcuing studies of human crossmodal attention and multisensory integration. In C. Spence \& J. Driver (Eds.), Crossmodal space and crossmodal attention (pp. 277-320). Oxford: Oxford University Press.

Spence, C., Nicholls, M. E. R., Gillespie, N., \& Driver, J. (1998). Cross-modal links in exogenous covert spatial orienting between touch, audition, and vision. Perception \& Psychophysics, 60, 544-557.

Spence, C., \& Parise, C. (2010). Prior-entry: A review. Consciousness and Cognition, 19, 364-379.

Spence, C., Shore, D. I., \& Klein, R. M. (2001). Multisensory prior entry. Journal of Experimental Psychology. General, 130, 799832. 
Stelmach, L. B., \& Herdman, C. M. (1991). Directed attention and perception of temporal order. Journal of Experimental Psychology. Human Perception and Performance, 17, 539-550.

Tassinari, G., \& Campara, D. (1996). Consequences of covert orienting to non-informative stimuli of different modalities: A unitary mechanism? Neuropsychologia, 34, 235-245.

van Damme, S., Gallace, A., Spence, C., Crombez, G., \& Moseley, G. L. (2009). Does the sight of physical threat induce a tactile processing bias? Modality-specific facilitation of attention induced by viewing threatening pictures. Brain Research, 1253, $100-106$.

van der Burg, E., Olivers, C. N. L., Bronkhorst, A. W., \& Theeuwes, J. (2008). Audiovisual events capture attention: Evidence from temporal order judgments. Journal of Vision, 8(5 (2)), 1-10.

van Eijk, R. L. J., Kohlrausch, A., Juola, J. F., \& van de Par, S. (2008). Audiovisual synchrony and temporal order judgments:
Effects of experimental method and stimulus type. Perception \& Psychophysics, 70, 955-968.

Vanderhaeghen, C., \& Bertelson, P. (1974). Limits of prior entryNonsensitivity of temporal order judgments to selective preparation affecting choice reaction-time. Bulletin of the Psychonomic Society, 4, 569-572.

Vatakis, A., Navarra, J., Soto-Faraco, S., \& Spence, C. (2008). Audiovisual temporal adaptation of speech: Temporal order versus simultaneity judgments. Experimental Brain Research, 185, 521-529.

Yates, M. J., \& Nicholls, M. E. R. (2009). Somatosensory prior entry. Attention, Perception, \& Psychophysics, 71, 847-859.

Zampini, M., Shore, D. I., \& Spence, C. (2005). Audiovisual prior entry. Neuroscience Letters, 381, 217-222.

Zhuang, X., \& Papathomas, T. V. (2009). Prior entry for feature-based attention: Are objects of the attended color perceived earlier [Abstract]. Journal of Vision, 9(8), 144a. 